\title{
Erythema Indicator
}

National Cancer Institute

\section{Source}

National Cancer Institute. Erythema Indicator. NCI Thesaurus. Code C154877.

An indication as to whether erythema is present. 